\title{
Chemical Analysis of Drinking Water Samples of Some Primary Schools from Magura District, Bangladesh
}

\author{
Md. Aminur Rahman ${ }^{1}$, Kamrun Nahar², Sharif Md. Al-Reza ${ }^{2, *}$ \\ ${ }^{1}$ Department of Public Health Engineering, Zonal Laboratory, Khulna, Bangladesh \\ ${ }^{2}$ Department of Applied Chemistry and Chemical Technology, Islamic University, Kushtia, Bangladesh
}

\section{Email address}

sharif@acct.iu.ac.bd (S. M. Al-Reza)

${ }^{*}$ Corresponding author

\section{To cite this article:}

Md. Aminur Rahman, Kamrun Nahar, Sharif Md. Al-Reza. Chemical Analysis of Drinking Water Samples of Some Primary Schools from Magura District, Bangladesh. Journal of Water Resources and Ocean Science. Vol. 5, No. 5, 2016, pp. 73-77.

doi: $10.11648 /$ j.wros.20160505.12

Received: August 6, 2016; Accepted: September 12, 2016; Published: October 10, 2016

\begin{abstract}
In order to ascertain water quality for human consumption, major and minor ions were evaluated in the drinking water supplied to the primary school students of Magura district in Bangladesh. Standard methods were used for determining physical and chemical characteristics of the water samples. Arsenic (As), Iron (Fe) and Manganese (Mn) contents of the drinking water samples were also analyzed by atomic absorption spectrophotometer (AAS). The data showed the variation of the investigated parameter in water samples as follows: pH 7.29 to 8.93, Electrical Conductivity (EC) 343 to $3000 \mu \mathrm{S} / \mathrm{cm}$, chloride 10 to $725 \mathrm{mg} / \mathrm{l}$, hardness 130 to $790 \mathrm{mg} / \mathrm{l}$ as $\mathrm{CaCO}_{3}$, Total Dissolved Solids (TDS) 178 to $1530 \mathrm{mg} / \mathrm{l}$, As Below the Detection Limit (BDL) to $0.012 \mathrm{mg} / \mathrm{l}, \mathrm{Fe} 0.03$ to $3.79 \mathrm{mg} / \mathrm{l}$ and $\mathrm{Mn} 0.03$ to $0.66 \mathrm{mg} / \mathrm{l}$. The concentrations of testing parameter in the drinking water samples were not permit all the sources of the World Health Organization (WHO) and Bangladesh Drinking Standards (BDS) water quality guideline values.
\end{abstract}

Keywords: Drinking Water, Primary School Students, AAS, Metal Ions, Water Quality

\section{Introduction}

From time immemorial, water assessment has always been a major concern. Today, the principal difficulty with which we are confronted is not so much access to water but more precisely the access to suitable water for drinking. Water can be the vehicle of a very high number of pathogenic agents voided into the external medium by the human or animal faeces and can thus be at the origin of many waterborne diseases. In 1996, WHO quantified to 4 billion, the number of diarrhea episodes which occurred in the world, and were responsible for the death of 3.1 million people of whom the large majority were children less than five years [1]. In the light of these figures, one realizes the importance of the problem of drinking water assessment and the capital need to seek solutions to improve the situation in this sector. The natural water analysis for physical, chemical properties including trace element contents are very important for public health studies especially for children. These studies are also a main part of pollution studies in the environment [2-4]. The 510 ages children are drinking water in the investigated water sources. The determinations in drinking water have been performed using classical analytical techniques including titrimetry, gravimetry and modern instrumental techniques such as atomic absorption spectrophotometry (AAS), inductively coupled plasma-mass spectrometry (ICP-MS), UV-Vis spectrometry, etc. Because of the low cost and easiness in usage, atomic absorption spectrophotometry is the main instrument for the determinations of the trace metal ions in drinking water in the analytical chemistry laboratories [5-7]. Every year approximately five thousand children are studying in these schools. Not only the children but also the local villagers were drinking water of these water sources (Tubewells). According to our literature review, no report has been published concerning the trace metal ions of these areas.

In the Present work arsenic, iron and manganese in drinking water samples from the water sources of different primary schools in Magura district were determined by atomic absorption spectrophotometer. Physical and chemical 
properties of the samples were also determined by using standard analytical methods.

\section{Materials and Method}

\subsection{Sample Collection}

The drinking water samples were collected in prewashed (with detergent, doubly de-ionised distilled water, diluted $\mathrm{HNO}_{3}$ and doubly de-ionised distilled water, respectively) high density polyethylene (HDPE) bottles from twenty different primary schools around Magura district in June 2013. The sampling locations were shown in Table-1. $\mathrm{pH}$ and electrical conductivity (EC) of the samples were measured while collecting the samples. Two liters (one liter for the determinations of main ions and one liter for metal determinations) of each water sample was taken in duplicate at two different sampling periods approximately 1 month apart. The determinations of the major ions of the water samples were performed within one week after sample collection.

The distances of laboratory from Magura district are approximately 40 killometers. The samples were obtained directly from the water pump after allowing the water to run for at least 25 minutes. The samples for metal determinations were filtered through a Millipore cellulose membrane of 0.45 $\mu \mathrm{m}$ pore size and were stored in 1 liter HDPE bottles and acidified to $1 \%$ with nitric acid. These samples were subsequently stored at $4{ }^{\circ} \mathrm{C}$ for as short a time as possible before analysis to minimize physicochemical changes.

Table 1. The locations of the collected water samples.

\begin{tabular}{|c|c|c|c|c|c|c|}
\hline \multirow{2}{*}{ SI No } & \multirow{2}{*}{ Upazilla } & \multirow{2}{*}{ Union } & \multirow{2}{*}{ Village } & \multirow{2}{*}{ Sampling Location } & \multicolumn{2}{|l|}{ GPS } \\
\hline & & & & & Latitude (N) & Longitude (E) \\
\hline 1 & MaguraSadar & BeroilPolita & Dighalkandi & $\begin{array}{l}\text { Dighalkandi Govt. } \\
\text { Primary School }\end{array}$ & $23^{\circ} 21^{\prime} 23^{\prime \prime}$ & $89^{\circ} 30^{\prime} 44^{\prime \prime}$ \\
\hline 2 & MaguraSadar & Chaulia & Chandpur & $\begin{array}{l}\text { ChandpurPurboparaRegd. } \\
\text { PrimarySchool }\end{array}$ & $23^{\circ} 27^{\prime} 06^{\prime \prime}$ & $89^{\circ} 29^{\prime} 12^{\prime \prime}$ \\
\hline 3 & MaguraSadar & Kuchiamora & Kuchiamora & $\begin{array}{l}\text { KuchiamoraGovt. } \\
\text { Primary School }\end{array}$ & $23^{\circ} 20^{\prime} 37^{\prime \prime}$ & $89^{\circ} 26^{\prime} 17^{\prime \prime}$ \\
\hline 4 & MaguraSadar & Atharokhada & Madhabpur & $\begin{array}{l}\text { Madhabpur Govt. } \\
\text { Primary School }\end{array}$ & $23^{\circ} 32^{\prime} 13^{\prime \prime}$ & $89^{\circ} 25^{\prime} 55^{\prime \prime}$ \\
\hline 5 & MaguraSadar & Moghi & TitarKhaPara & $\begin{array}{l}\text { TitarKhapara Regd. } \\
\text { Primary School }\end{array}$ & $23^{\circ} 25^{\prime} 28^{\prime \prime}$ & $89^{\circ} 23^{\prime} 16^{\prime \prime}$ \\
\hline 6 & Mohammadpur & Binodpur & Kalukandi & $\begin{array}{l}\text { Kalukandi Regd. Govt. } \\
\text { Primary School }\end{array}$ & $23^{\circ} 26^{\prime} 34^{\prime \prime}$ & $89^{\circ} 32^{\prime} 12^{\prime \prime}$ \\
\hline 7 & Mohammadpur & Balidia & Charborboria & $\begin{array}{l}\text { Charbororia Regd. } \\
\text { Primary School }\end{array}$ & $23^{\circ} 22^{\prime} 56^{\prime \prime}$ & $89^{\circ} 34^{\prime} 02^{\prime \prime}$ \\
\hline 8 & Mohammadpur & $\begin{array}{l}\text { Mohammadpu } \\
\mathrm{r}\end{array}$ & Raipur & $\begin{array}{l}\text { Raipur Regd. } \\
\text { Primary School }\end{array}$ & $23^{\circ} 25^{\prime} 49^{\prime \prime}$ & $89^{\circ} 35^{\prime} 19^{\prime \prime}$ \\
\hline 9 & Mohammadpur & Polashbaria & Bathari & $\begin{array}{l}\text { Bathari Regd. } \\
\text { Primary School }\end{array}$ & $23^{\circ} 20^{\prime} 08^{\prime \prime}$ & $89^{\circ} 33^{\prime} 21^{\prime \prime}$ \\
\hline 10 & Mohammadpur & Rajapur & Rajgonj & $\begin{array}{l}\text { Rajgonj Govt. } \\
\text { Primary School }\end{array}$ & $23^{\circ} 23^{\prime} 13^{\prime \prime}$ & $89^{\circ} 31^{\prime} 12^{\prime \prime}$ \\
\hline 11 & Salikha & Gangarampur & Bamonkhali & $\begin{array}{l}\text { Bamonkhali Govt. } \\
\text { Primary School }\end{array}$ & $23^{\circ} 15^{\prime} 34^{\prime \prime}$ & $89^{\circ} 26^{\prime} 43^{\prime \prime}$ \\
\hline 12 & Salikha & $\begin{array}{l}\text { Dhaneswargat } \\
\text { i }\end{array}$ & Dhaneswargati & $\begin{array}{l}\text { Dhaneswargati Community. } \\
\text { PrimarySchool }\end{array}$ & $23^{\circ} 25^{\prime} 17^{\prime \prime}$ & $89^{\circ} 18^{\prime} 15^{\prime \prime}$ \\
\hline 13 & Salikha & $\begin{array}{l}\text { Dhaneswargat } \\
\text { i }\end{array}$ & Tilkhari & $\begin{array}{l}\text { Tilkhari Govt. } \\
\text { Primary School }\end{array}$ & $23^{\circ} 22^{\prime} 56^{\prime \prime}$ & $89^{\circ} 17^{\prime} 09^{\prime \prime}$ \\
\hline 14 & Salikha & Talkhari & Chandra & $\begin{array}{l}\text { Chandra Govt. } \\
\text { Primary School }\end{array}$ & $23^{\circ} 21^{\prime} 03^{\prime \prime}$ & $89^{\circ} 18^{\prime} 12^{\prime \prime}$ \\
\hline 15 & Salikha & Satakhali & Kholabaria & $\begin{array}{l}\text { KholabariaRegd. } \\
\text { Primary School }\end{array}$ & $23^{\circ} 18^{\prime} 32^{\prime \prime}$ & $89^{\circ} 19^{\prime} 52^{\prime \prime}$ \\
\hline 16 & Sreepur & Sreekol & Sreekol & $\begin{array}{l}\text { Sreekol Govt. } \\
\text { Primary School }\end{array}$ & $23^{\circ} 36^{\prime} 49^{\prime \prime}$ & $89^{\circ} 21^{\prime} 44^{\prime \prime}$ \\
\hline 17 & Sreepur & Goespur & Joka & $\begin{array}{l}\text { Joka Govt. } \\
\text { PrimarySchool }\end{array}$ & $23^{\circ} 36^{\prime} 41^{\prime \prime}$ & $89^{\circ} 22^{\prime} 35^{\prime \prime}$ \\
\hline 18 & Sreepur & Amolsar & Kodla & $\begin{array}{l}\text { Kodla Govt. } \\
\text { PrimarySchool }\end{array}$ & $23^{\circ} 39^{\prime} 05^{\prime \prime}$ & $89^{\circ} 25^{\prime} 58^{\prime \prime}$ \\
\hline 19 & Sreepur & Dariapur & Char Chougachi & $\begin{array}{l}\text { Charchougachi Regd. } \\
\text { Primary School }\end{array}$ & $23^{\circ} 37^{\prime} 59^{\prime \prime}$ & $89^{\circ} 27^{\prime} 22^{\prime \prime}$ \\
\hline 20 & Sreepur & Kadirpara & Gashiara & $\begin{array}{l}\text { Gashiara Govt. } \\
\text { Primary School }\end{array}$ & $23^{\circ} 33^{\prime} 32^{\prime \prime}$ & $89^{\circ} 29^{\prime} 14^{\prime \prime}$ \\
\hline
\end{tabular}

\subsection{Reagent and Solutions}

Analytical grade reagent chemicals were employed for the preparation of all solutions. Freshly prepared double de- ionised distilled water, from a quartz still, was used in all experiments. Hydrochloric acid (5M), Sodium Borohydride reagent $\left(0.6 \% \mathrm{NaBH}_{4}\right.$ solution), Potassium Iodide $(20 \% \mathrm{KI})$ solution as a reductant, Inert gas Argon (as a carrier gas) for 
HVG system (determination of As), Air-Acetylene as a fuel gas for direct flame system (determination of $\mathrm{Fe}$ and $\mathrm{Mn}$ ), Commercial grade Standard solutions(CRM) of As, Fe, Mn solutions were used throughout the experiments.

\subsection{Apparatus}

Prior to analysis, all instruments were calibrated according to manufacturer's recommendations. $\mathrm{pH}$ was measured by using SensIONTM-MM340 digital meter. Conductivity was determined using an Electrical Conductivity meter CM-21 P. The meter was calibrated by using standard $\mathrm{EC}=1214 \mu \mathrm{s} / \mathrm{cm}$. Chloride was determined using the Argentometric Method. Determination of hardness was done by EDTA titrimetric methods. Atomic absorption spectrometer (ShimadzuAA7000) equipped with deuterium background correction, double beam system were used for the analysis of Arsenic (Hydride Vapour Generated Method), Iron and Manganese (direct flame method). All examine were conducted according to American Public Health Association Standard Methods [8].

\subsection{Analysis for TDS and Metal Ions}

Total Dissolved Solids were analyzed by the use of multimeter using respective standards solutions. Arsenic, Iron \& Manganese were analyzed by Atomic Absorption Spectrophotometric Method. As(V) is reduced to As(III) using potassium iodide and sodiumborohydride reagent to form arsine vapour and detect the total arsenic. Here inert gas argon is used as a carrier gas. This process is called Hydride Vapour Generation (HVG). On the other hand iron \& manganese is analyzed by atomization process creating a flame by the combustion of air \& acetylene gas (flame temperature nearly about $2200^{\circ} \mathrm{C}$ ).

\section{Results and Discussion}

\subsection{Physical and Chemical Properties of the Samples}

The main physical and chemical properties of the drinking water samples including $\mathrm{pH}$, electrical conductivity, chloride, hardness and Total Dissolved Solids (TDS) from Magura districts were given in Table-2. The $\mathrm{pH}$ values in the range of 7.29 to 8.93 (lowest in Gashiara Govt. Primary School, highest Charborboria Regd. Primary School). The ranges for electrical conductivity were 343 to $3000 \mu \mathrm{S} / \mathrm{cm}$. The lowest level of the chloride in Raipur Regd. Primary School and Chandra Govt. Primary School as 10mg/l, the highest level of the chloride was found in Bathari Registard Primary School as $725 \mathrm{mg} / \mathrm{l}$. The hardness of the samples was in the ranges of $130 \mathrm{mg} / \mathrm{l}$ to $790 \mathrm{mg} / \mathrm{l}$ as $\mathrm{CaCO}_{3}$. The Total Dissolved Solids (TDS) of the samples were in the range of 178 to 1530 $\mathrm{mg} / \mathrm{l}$. The drinking water quality standard guideline of hardness in Bangladesh is $500 \mathrm{mg} / \mathrm{l}$ as $\mathrm{CaCO}_{3}$ and Total Dissolved Solids (TDS) is $1000 \mathrm{mg} / \mathrm{l}$. Dighalkandi Govt. Primary School and Bathari Regd. Primary School does not permitted these two parameter. The Bangladesh Drinking Standards (BDS) for $\mathrm{pH}$ is 6.5-8.5 whereas Chloride is between $150-600 \mathrm{mg} / \mathrm{l}$. No standard guideline value is proposed for Electrical Conductivity .

\subsection{Trace Metal Ions}

The drinking water samples collected from the twenty water points in Magura were analyzed by atomic absorption spectrometry in triplicate to determine arsenic, iron and manganese. The concentrations are given in Table-3.

The lowest level of Arsenic (BDL) was detected in Bathari Regd. Primary School, Mohammadpur Upazilla and the highest level in Kalukandi Regd. Govt. Primary School as $0.012 \mathrm{mg} / \mathrm{l}$. Bangladesh standards for drinking water quality of arsenic is $0.05 \mathrm{mg} / \mathrm{l}$ but WHO guideline is $0.01 \mathrm{mg} / \mathrm{l}$. So, as can be seen in Table-3, in the locations Kalukandi Regd. Govt. Primary School and Charchougachi Regd. Primary School permitted BDS guideline value but does not WHO guideline [1].

Table 2. The physical and chemical properties of drinking water samples.

\begin{tabular}{|c|c|c|c|c|c|c|c|c|}
\hline $\begin{array}{l}\text { SI } \\
\text { No }\end{array}$ & $\begin{array}{l}\text { Type of } \\
\text { Tube-wells }\end{array}$ & $\begin{array}{l}\text { Deepth } \\
\text { (m) }\end{array}$ & Sampling Location & pH & $\begin{array}{l}\text { EC } \\
(\mu \mathrm{s} / \mathrm{cm})\end{array}$ & $\begin{array}{l}\text { Chloride } \\
(\mathrm{mg} / \mathrm{l})\end{array}$ & $\begin{array}{l}\text { Hardness } \\
\left(\mathrm{mg} \mathrm{CaCO}_{3} / \mathrm{l}\right)\end{array}$ & $\begin{array}{l}\text { TDS } \\
(\mathrm{mg} / \mathrm{l})\end{array}$ \\
\hline 1 & Tara Deep & 164.63 & $\begin{array}{l}\text { Dighalkandi Govt. } \\
\text { Primary School }\end{array}$ & 7.70 & 3000 & 517 & 790 & 1530 \\
\hline 2 & Tara Deep & 167.68 & $\begin{array}{l}\text { Chandpur Purbopara Regd. } \\
\text { Primary School }\end{array}$ & 8.41 & 496 & 20 & 220 & 258 \\
\hline 3 & Tara Deep & 167.68 & $\begin{array}{l}\text { Kuchiamora Govt. } \\
\text { Primary School }\end{array}$ & 8.03 & 851 & 64 & 260 & 443 \\
\hline 4 & Tara Deep & 163.10 & $\begin{array}{l}\text { Madhabpur Govt. } \\
\text { Primary School }\end{array}$ & 8.52 & 457 & 40 & 210 & 238 \\
\hline 5 & Tara Deep & 164.63 & $\begin{array}{l}\text { Titar Khapara Regd. } \\
\text { Primary School }\end{array}$ & 8.70 & 367 & 15 & 210 & 191 \\
\hline 6 & Tara Deep & 149.28 & $\begin{array}{l}\text { Kalukandi Regd. Govt. } \\
\text { Primary School }\end{array}$ & 8.62 & 402 & 15 & 190 & 210 \\
\hline 7 & Tara Deep & 171.28 & $\begin{array}{l}\text { Charbororia Regd. } \\
\text { Primary School }\end{array}$ & 8.93 & 1030 & 144 & 150 & 536 \\
\hline 8 & Tara Deep & 167.28 & $\begin{array}{l}\text { Raipur Regd. } \\
\text { Primary School }\end{array}$ & 8.76 & 343 & 10 & 130 & 178 \\
\hline 9 & Tara Deep & 159.28 & Bathari Regd. & 8.15 & 2520 & 725 & 600 & 1310 \\
\hline
\end{tabular}




\begin{tabular}{|c|c|c|c|c|c|c|c|c|}
\hline $\begin{array}{l}\text { SI } \\
\text { No }\end{array}$ & $\begin{array}{l}\text { Type of } \\
\text { Tube-wells }\end{array}$ & $\begin{array}{l}\text { Deepth } \\
\text { (m) }\end{array}$ & Sampling Location & pH & $\begin{array}{l}\text { EC } \\
(\mu \mathrm{s} / \mathrm{cm})\end{array}$ & $\begin{array}{l}\text { Chloride } \\
\text { (mg/l) }\end{array}$ & $\begin{array}{l}\text { Hardness } \\
\left(\mathrm{mg} \mathrm{CaCO}_{3} / \mathrm{l}\right)\end{array}$ & $\begin{array}{l}\text { TDS } \\
(\mathrm{mg} / \mathrm{l})\end{array}$ \\
\hline 10 & Tara Deep & 153.28 & $\begin{array}{l}\text { Primary School } \\
\text { Rajgonj Govt. } \\
\text { Primary School }\end{array}$ & 8.30 & 686 & 25 & 250 & 357 \\
\hline 11 & Tara Deep & 135.67 & $\begin{array}{l}\text { Bamonkhali Govt. } \\
\text { Primary School }\end{array}$ & 8.72 & 993 & 129 & 210 & 516 \\
\hline 12 & Tara Deep & 150.91 & $\begin{array}{l}\text { Dhaneswargati Community. } \\
\text { Primary School }\end{array}$ & 8.87 & 424 & 45 & 210 & 220 \\
\hline 13 & Tara Deep & 166.15 & $\begin{array}{l}\text { Tilkhari Govt. } \\
\text { Primary School }\end{array}$ & 8.71 & 440 & 25 & 210 & 229 \\
\hline 14 & Tara Deep & 149.39 & $\begin{array}{l}\text { Chandra Govt. } \\
\text { Primary School }\end{array}$ & 8.62 & 348 & 10 & 160 & 180 \\
\hline 15 & Tara Deep & 160.06 & $\begin{array}{l}\text { Kholabaria Regd. } \\
\text { Primary School }\end{array}$ & 8.57 & 549 & 20 & 250 & 285 \\
\hline 16 & Tara Deep & 179.87 & $\begin{array}{l}\text { Sreekol Govt. } \\
\text { Primary School }\end{array}$ & 8.12 & 877 & 89 & 280 & 456 \\
\hline 17 & Tara Deep & 164.63 & $\begin{array}{l}\text { Joka Govt. } \\
\text { Primary School }\end{array}$ & 7.79 & 1212 & 141 & 440 & 630 \\
\hline 18 & Tara Deep & 161.58 & $\begin{array}{l}\text { Kodla Govt. } \\
\text { Primary School }\end{array}$ & 8.22 & 848 & 103 & 280 & 440 \\
\hline 19 & Tara Deep & 158.53 & $\begin{array}{l}\text { Charchougachi Regd. } \\
\text { Primary School }\end{array}$ & 8.15 & 593 & 19 & 220 & 308 \\
\hline 20 & Tara Deep & 158.53 & $\begin{array}{l}\text { Gashiara Govt. } \\
\text { Primary School }\end{array}$ & 7.29 & 1042 & 76 & 410 & 542 \\
\hline
\end{tabular}

The highest iron level was found in Tilkhari Govt. Primary Schoolin Salikha Upazillaas $3.79 \mathrm{mg} / \mathrm{l}$ and lowest in Rajgonj Govt. Primary Schoolas $0.03 \mathrm{mg} / \mathrm{l}$ in Mohammadpur Upazilla. Regarding iron, it can be seen that maximum water points (twelve sources out of twenty-60\%) exceeded the Bangladesh Drinking Standards as well as WHO Standards [1], which agree with results obtained by other authorsin other countries [9-11]. The BDS of iron is $0.3-1.0 \mathrm{mg} / \mathrm{l}$.
The highest manganese concentration was detected in Gashiara Govt. Primary School in Sreepur upazilla as 0.66 $\mathrm{mg} / \mathrm{l}$ and lowest in Kalukandi Regd. Govt. Primary School as $0.03 \mathrm{mg} / \mathrm{l}$ in Mohammadpur upazilla. The acceptable limit of Manganese in Bangladesh is $0.1 \mathrm{mg} / \mathrm{l}$. So, eight water points out of twenty $(40 \%)$ containing higher than the BDS guideline value.

Table 3. The concentrations of trace metal ions in drinking water samples.

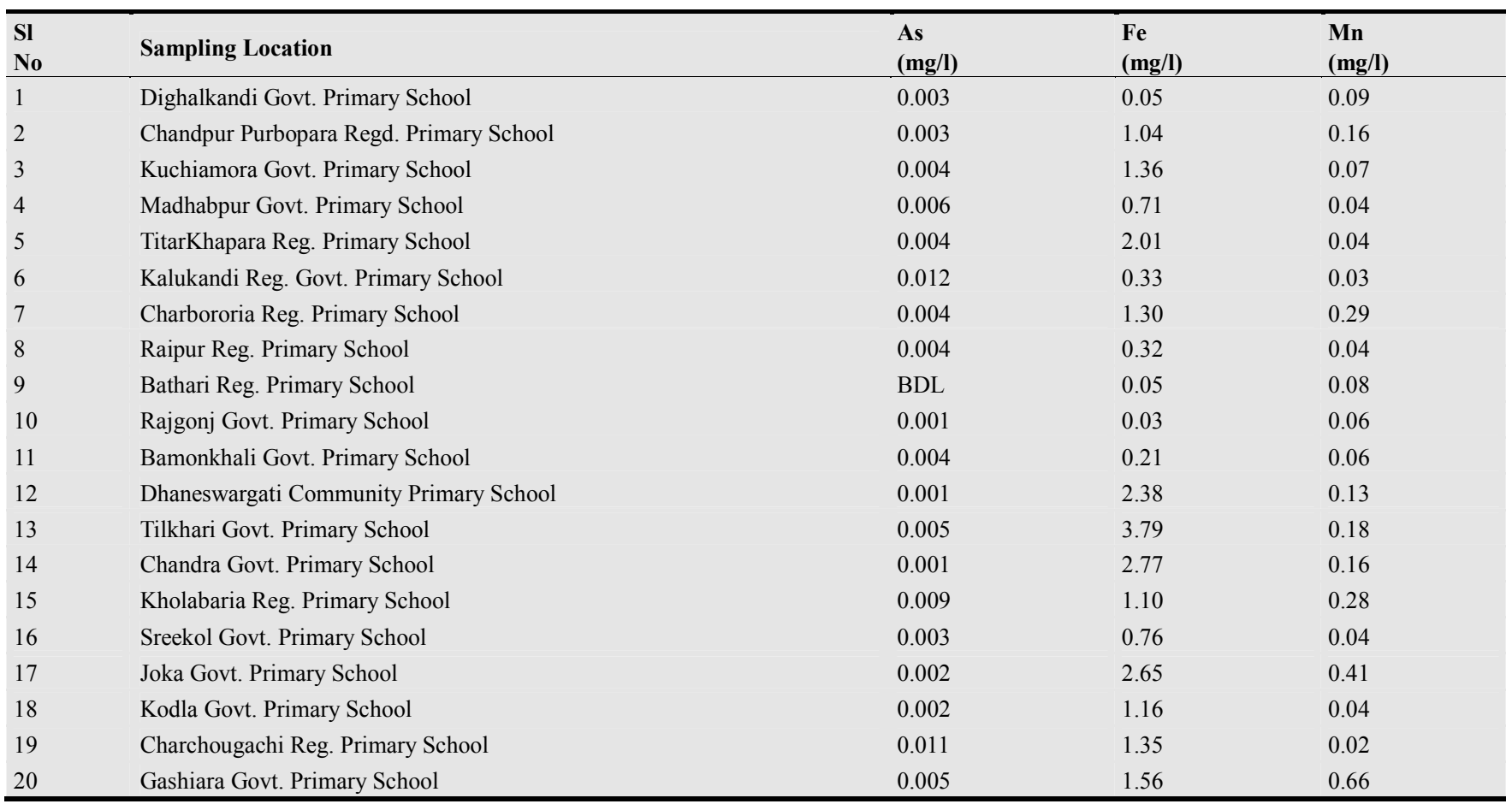

BDL: Below the detection limit. 


\section{Conclusion}

From the above discussion we can conclude that the people of Bangladesh are living with a danger of drinking water. Hence we need to adapt steps to cope the problems. Being a responsible citizen of Bangladesh we should make awareness to the people about harmfulness of heavy metals present in drinking water and inspire community people about sharing of safe drinking water.

\section{References}

[1] World Health Organization (WHO) (2008). Guidelines for Drinking-Water Quality, Third Edition, Incorporating the First and Second Addenda, Volume 1: Recommendations, WHO, Geneva.

[2] Bakraji, E, H., Karajo, J. (1999). Determination of heavy metals in Damascus drinking water using total reflection Xray fluorescence. Water Quality Res. J. Canada, 34: 305-310.

[3] Zereen, F., Islam, F., Habib, M, A., Begum, D, A., Zaman, M, S. (2000). Inorganic pollutant in the Padma river, Bangladesh. Environmental Geology, 39: 1059-1062.

[4] Dogan, M., Soylak, M. (2002). Determination of some trace elements in mineral spring waters by Total reflection X-Ray fluorescence Spectrometry (TXRF), J. Trace Microprobe Techn., 20(2): 261-268.

[5] Basset, J., Denney, T, C. (1983). Vogel's Textbook of quantitative inorganic analysis, Longman, London.

[6] Ballinger, D, G. (1989). Methods for chemical analysisof water and wastes, EPA, Ohio, USA.

[7] Soylak, M., Elci, L., Dogan, M. (1992). Kayseri cevresindeki akarsularda bazi katyon ve anyonlarin tayini, Marmara Universitesi Fen Bilimleri Dergisi, 9: 85-99.

[8] American Public Health Association (APHA), (1999). Standard Methods for the Examination of Water and Wastewater.

[9] Latino, J, C., Sears, D, C., Portala, F., Shutter, I, L. (1995). The simultaneous determination of dissolved $\mathrm{Ag}, \mathrm{Cd}, \mathrm{Pb}$ and $\mathrm{Sb}$ in potable waters by ETAAS, Atom. Spectrosc, 16:121126.

[10] Mahmood, S., Naeem, N., Siddiqui, S, I., Khan, F, A., (1998). Metal contamination in ground water of Korangi Industrial Area, Karachi. J. Chem. Soc. Pakistan, 20: 125-131.

[11] Asubiojo, O, I., Nkono, N, A., Ogunsua, O, A., Oluwole, A, F., Ward, N, I., Akanle, O, A., Spyrou, N, M. (1997). Trace elements in drinking and ground water samples in Southern Nigeria, Sci. Total Environ, 208: 1-8. 